\title{
Cellulose acetate, a new candidate feed supplement for ruminant animals: In vitro evaluations
}

\author{
Y. Watabe, ${ }^{*}$ Y. Suzuki, ${ }^{*}$ S. Koike, ${ }^{*}$ S. Shimamoto, $†$ and Y. Kobayashi ${ }^{* 1}$ \\ ${ }^{*}$ Graduate School of Agriculture, Hokkaido University, Sapporo 060-8589, Japan \\ †Daicel Corporation, Tokyo Head Office, Tokyo 108-8230, Japan
}

\section{ABSTRACT}

Cellulose acetate (CA), a derivative of cellulose in which some hydroxyl groups are substituted with acetyl groups, was evaluated as a new cellulosic feed source for ruminants. In the present work, a series of in vitro studies was carried out to determine how CA supplementation affects rumen fermentation and microbiota. Batch culture studies were conducted to select the type of CA suitable for feed use and to define the optimal supplementation level. Rumen fluid from 2 Holstein cows was mixed with McDougall's buffer in test tubes into which grass hay and concentrate containing a fiber source [cellulose (control), water-soluble CA (WSCA), or insoluble CA] had been placed. Each fiber source was supplemented at $10 \%$ of total substrate. Tubes were incubated for $24 \mathrm{~h}$ to determine fermentation and microbial parameters. Then, the dose response of these parameters to different supplementation levels of WSCA $(0,7.5,15,22.5$, and $30 \%)$ was tested in the same manner. We also operated a continuous culture system with WSCA supplementation and evaluated the effects on digestibility, fermentation, and microbial parameters. The supplementation level of WSCA was set at $15 \%$ of total feed. In batch culture studies, WSCA, but not insoluble CA, yielded dose-dependent increases in ruminal acetate levels. In the continuous culture system study, WSCA yielded increases in ruminal acetate levels and in the abundance of bacteria of the genus Prevotella, including Prevotella ruminicola. Dry matter digestibility and total gas production were not affected. These results suggest that WSCA supplementation at $15 \%$ of total feed yielded increased acetate levels without negatively affecting feed digestion; these effects may reflect activation of Prevotella species. As ruminal acetate is involved in milk fat synthesis, WSCA can

Received April 23, 2018

Accepted August 16, 2018.

${ }^{1}$ Corresponding author: kyas@anim.agr.hokudai.ac.jp be considered as a candidate feed additive suitable for dairy cattle.

Key words: cellulose acetate, Prevotella, rumen fermentation, rumen microbiota

\section{INTRODUCTION}

Cellulose acetate $(\mathbf{C A})$ is a semisynthetic polymer obtained through the esterification of acetic acid with cellulose, resulting in substitution of acetyl groups for some of the hydroxyl groups of cellulose. The degree of substitution (DS) changes the physical properties of CA, including water solubility and viscosity (Samios et al., 1997; Teramoto, 2015; Zhou et al., 2016). High DS makes CA less soluble in water, whereas a limited range of DS (0.5-1.1) renders CA water soluble (Miyamoto et al., 1985; Shimamoto et al., 2014). These characteristics have made CA attractive for industrial applications, including the widespread use of this cellulose ester as a feedstock for the production of textiles, cigarette filters, and other materials (Puls et al., 2011; Robertson et al., 2012; Lalia et al., 2013). In fact, CA is considered a renewable, biodegradable, nontoxic material with decreased flammability and cost compared with cellulose itself (Gaana et al., 2011; Fei et al., 2015). Global industry analysis projected a worldwide market for CA of approximately 1.05 million tonnes by 2017 (Candido and Gonçalves, 2016).

For further application of CA, we have been focusing on the possible use of $\mathrm{CA}$ as a feed for ruminant animals, which are able to ferment cellulosic materials to use VFA as an energy source. The VFA generated via ruminal fermentation contribute to ruminant nutrition, providing up to $70 \%$ of these animals' maintenance energy requirements (Bergman, 1990). This conversion is enabled by the presence of a dense and diverse rumen microbial population that is capable of degrading plant materials in which cellulose and other cell wall components constitute the main carbohydrate source. The ruminal microbial population encodes many different enzymatic systems (Flint, 1997), several of which 
are expected to contribute to the metabolism of CA to produce VFA and support animal nutrition.

Cellulose acetate is degraded by several bacterial enzymes (Puls et al., 2011; Biely, 2012). For instance, 2 strains of the soil bacterium Neisseria sicca have been shown to degrade CA and release acetate (Sakai et al., 1996; Moriyoshi et al., 2003). Separately, Bacillus sp. strain S2055 has been shown to be capable of degrading CA to yield acetate (Ishigaki et al., 2000). If this acetate is generated from the acetyl groups of $\mathrm{CA}$, debranching enzymes such as acetyl esterase are expected to have an important role in the degradation of CA. The presence of such debranching enzymes in certain species of rumen microbes has been described, and the importance of these proteins in the fermentation of complex polysaccharides has been emphasized in rumen fermentation (Flint, 1997).

Based on these observations, we hypothesized that CA could modify the rumen microbial population and associated fermentative processes, resulting in an increase in acetate production. Acetate is the major VFA used to support the maintenance of host animals and is known to be utilized for milk fat synthesis in the mammary gland of dairy cows (Urrutia and Harvatine, 2017). Therefore, if ruminal acetate production is promoted through CA supplementation, CA is expected to be a new candidate feed or additive for the promotion of energy supply in host animals while also potentially contributing to the prevention of milk fat depression in dairy cows under heat stress conditions (Bouraoui et al., 2002), as is expected to occur with global warming and roughage intake decrease. In the present study, the potential use of CA as a ruminant feed was evaluated by conducting a series of in vitro culture studies.

\section{MATERIALS AND METHODS}

\section{Substrates and Rumen Fluid}

Two types of CA powder $(60-200 \mu \mathrm{m}$ in nominal particle size), water-soluble CA (WSCA; WSCA80, Daicel, Osaka, Japan) and insoluble CA (ISCA; CDA-093, Daicel), were used in the present study. The DS by acetyl groups was 0.78 for WSCA and 2.4 for ISCA. The degree of polymerization was 124 and 301 for WSCA and ISCA, respectively. These CA reagents were obtained as products of Daicel. As a control substrate, cellulose powder (Asahi Chemical Industry Co. Ltd., Osaka, Japan) was used. This was a microcrystalline cellulose with 100 to 300 degree of polymerization and $50-\mu \mathrm{m}$ nominal particle size. Feeds such as grass hay and commercial concentrate were used as basic substrates as fed to rumen fluid donor cows.
Rumen content for batch and continuous cultures was collected from 2 rumen-fistulated Holstein dry cows fed a timothy hay and concentrate (Monster 18; Mercian, Tokyo, Japan) diet (in a 2:1 ratio) at the experimental farm of Hokkaido University (Sapporo, Japan). The timothy hay contained $13 \% \mathrm{CP}, 50 \% \mathrm{NDF}$, and $60 \%$ TDN, and the commercial formula feed contained $13 \%$ $\mathrm{CP}$ and $76 \%$ TDN on a DM basis. Equal volumes of rumen content from each cow were combined and placed in a bottle flushed with $\mathrm{N}_{2}$ gas; the resulting mixture was transferred to the laboratory within $30 \mathrm{~min}$. The rumen content was strained through 2 layers of surgical gauze and used for the following in vitro experiments. All procedures were approved by the Animal Care and Welfare Committee of Hokkaido University.

\section{Batch Culture}

In vitro batch culture tests were performed as follows. Artificial saliva (McDougall, 1948) and strained rumen fluid were combined in a 1:1 ratio ( $\mathrm{vol} / \mathrm{vol}$ ), and a $10-\mathrm{mL}$ aliquot of the mixture was transferred to a test tube (180 $\mathrm{mm}$ length, $10 \mathrm{~mm}$ diameter) in which a mixture of $0.12 \mathrm{~g}$ of grass hay and $0.06 \mathrm{~g}$ of concentrate had been placed. The nutrient composition of each feed placed was identical to that fed to the respective rumen content donors. For determination of the more potent CA type, the dose of each fiber source (cellulose, WSCA, or ISCA) was set at $10 \%$ of total substrate on a DM basis (i.e., $0.02 \mathrm{~g}$ of each cellulose was added to $0.18 \mathrm{~g}$ of hay and concentrate). For the dose-response assays, supplementation levels of $0,7.5$, $15,22.5$, and $30 \%$ were tested in the same manner. The headspace of each tube was flushed with $\mathrm{N}_{2}$ gas and sealed with a butyl rubber stopper and plastic cap; the tubes were then incubated at $39^{\circ} \mathrm{C}$ for $24 \mathrm{~h}$. Each reaction was performed in quadruplicate $(\mathrm{n}=4)$. After incubation, total gas production was measured through a needle-attached pressure gauge $(\mathrm{A} \phi 60 \mathrm{~B}, \mathrm{GL}$ Sciences, Tokyo, Japan), and gas samples were analyzed for $\mathrm{H}_{2}, \mathrm{CH}_{4}$, and $\mathrm{CO}_{2}$ using $\mathrm{GC}$ (Watanabe et al., 2010). Culture fluids were used for VFA and microbial population analyses. These batch culture experiments were carried out to determine the more potent form of CA (water-soluble vs. insoluble) in terms of improvement in VFA production and to evaluate the dose level of the selected CA (WSCA; see Results) suitable for such improvement.

\section{Continuous Culture}

The rumen simulation technique (RUSITEC; Czerkawski and Breckenridge, 1977) was used to evalu- 
ate longer term rumen responses to WSCA as the selected material. The fermentation system was equipped with 8 fermentors, each of which had a $650-\mathrm{mL}$ working capacity. The procedure for operation, including feeding and sampling, was as described by Watanabe et al. (2010). Strained and pooled rumen fluid from 2 cows was mixed with artificial saliva at an 8:5 ratio and used as the rumen inoculum. Incubation lasted $7 \mathrm{~d}$, consisting of $5 \mathrm{~d}$ for adaptation and $2 \mathrm{~d}$ for sampling. Artificial saliva ( $\mathrm{pH}$ 6.8) was continuously supplied using a peristaltic pump with a dilution rate of $0.5 \mathrm{vol} / \mathrm{d}$. The experimental diet, a ground mixture consisting of $9 \mathrm{~g}$ of grass hay and $3 \mathrm{~g}$ of concentrate (the same as that fed to the donor cows), was fed by nylon bag to each fermentor every $24 \mathrm{~h}$. The 8 fermentors were divided into 2 groups (4 fermentors per group), to which 5.1 $\mathrm{g}$ of cellulose (control) or $2.55 \mathrm{~g}$ of WSCA and 2.55 $\mathrm{g}$ of cellulose (treatment) was supplemented at feeding time (directly to each fermentor but not into the nylon bag). Thus, the dose level of WSCA was $15 \%$ of the total feed. Each nylon bag was taken from the fermentor after $48 \mathrm{~h}$ of incubation and rinsed with 20 $\mathrm{mL}$ of artificial saliva to recover microbes adhering to feed particles. The artificial saliva used for rinsing was returned to the fermentor at each feeding time. Rumen fluid samples were recovered at 3 -h intervals from each fermentor, with sampling performed directly through a pipette. Feeding, rinsing of the bags, and pipette-aided sampling were performed under $\mathrm{CO}_{2}$ flushing to maintain an anaerobic atmosphere. Ruminal $\mathrm{pH}$, VFA, and ammonia were analyzed using all individual samples taken at 3-h intervals. Disappearance of DM was determined by analyzing feed residues in nylon bag samples recovered before feeding for the last $2 \mathrm{~d}$. The microbial population was analyzed using culture samples obtained before feeding for the last $2 \mathrm{~d}$. Fermentation gas was collected continuously in a plastic bag (Tedlar PDF; DuPont, Wilmington, DE) connected to each fermentor for the last $2 \mathrm{~d}$. Total volume of gas produced was measured by spirometer (Sanshin Kogyo, Tokyo, Japan), and gas composition was analyzed by CG (Shimadzu, Kyoto, Japan).

\section{Chemical Analysis}

Gases $\left(\mathrm{H}_{2}, \mathrm{CH}_{4}\right.$, and $\left.\mathrm{CO}_{2}\right)$ from microbial fermentation in batch cultures and RUSITEC were analyzed using a GC-8A gas chromatograph (Shimadzu) equipped with parallel columns of Porapak Q (Waters, Milford, MA) and Molecular Sieve 13X (Restek, Bellefonte, PA) and a thermal conductivity detector. We analyzed VFA as described below. Briefly, culture fluid was mixed with $25 \%$ meta-phosphoric acid at a 5:1 ratio, incubated overnight at $4^{\circ} \mathrm{C}$, and centrifuged at $10,000 \times g$ at $4^{\circ} \mathrm{C}$ for 10 min. Following the addition of crotonic acid as an internal standard, the supernatant was injected into a GC-14B gas chromatograph (Shimadzu) equipped with an ULBON HR-20M fused silica capillary column (0.53 $\mathrm{mm}$ i.d. $\times 30 \mathrm{~m}$ length, 3.0- $\mu \mathrm{m}$ film; Shinwa, Kyoto, Japan) and a flame-ionization detector. Ammonia nitrogen concentration was spectrophotometrically determined using the indophenol reaction (Weatherburn, 1967).

\section{Microbial Analysis}

We recovered samples for microbiological analysis from each fermentor fluid at the time of withdrawal of the nylon bag (after rinsing but before feeding with a new nylon bag), so that both planktonic and feedassociated microbes were included. These samples were immediately frozen and held at $-80^{\circ} \mathrm{C}$. Extraction of DNA for microbial analysis was performed using the repeated bead beating plus column method described by $\mathrm{Yu}$ and Morrison (2004). The resulting DNA was used for real-time PCR to quantify the abundance of rumen representatives, including total bacteria, total archaea, Fibrobacter succinogenes, Ruminococcus albus, Ruminococcus flavefaciens, genus Prevotella, Prevotella bryantii, Prevotella ruminicola, Anaerovibrio lipolytica, Megasphaera elsdenii, Ruminobacter amylophilus, Selenomonas ruminantium, Streptococcus bovis, Succinivibrio dextrinosolvens, genus Treponema, Treponema bryantii, and Butyrivibrio fibrisolvens. All quantitative PCR (qPCR) details (see Supplemental Table S1; https://doi.org/10.3168/jds.2018-14969) such as primers, standards, PCR conditions, and calculations were as described by Koike et al. (2007). In brief, a standard plasmid containing the respective target gene sequence was obtained by PCR cloning using a target-specific primer set. The abundance of each standard plasmid was calculated using the molecular weight of nucleic acid and the length (in base pairs) of the cloned standard plasmid as described by Koike et al. (2007). A LightCycler system and a KAPA SYBR Fast qPCR Kit (Kapa Biosystems, Charlestown, MA) were used with 10-fold serial dilutions of standard plasmid for the respective target (16S rRNA gene or $18 \mathrm{~S}$ rRNA gene sequence specific to each target microbe). We calculated microbial quantity using amplification curves obtained from both standard and sample. The specificity of PCR amplification was confirmed using melting curve analysis of the PCR products by increasing the temperature from $70^{\circ} \mathrm{C}$ to $95^{\circ} \mathrm{C}$ at a rate of $0.1^{\circ} \mathrm{C} / \mathrm{s}$. Microbial abundance was determined as absolute abundance of rRNA genes for total bacteria or by relative proportion in total bacterial abundance for total archaea and specific bacteria. 
To comprehensively analyze the microbial community, we used DNA samples at the 0-h feeding in each fermentor of RUSITEC for MiSeq analysis (Illumina Inc., San Diego, CA). Sequencing was performed by Hokkaido System Science Co. Ltd. (Sapporo, Japan). The V3 to V4 regions of the $16 \mathrm{~S}$ rRNA gene or $18 \mathrm{~S}$ rRNA gene were amplified using 2-primer sets: S-D-Bact-0341-b-S-17 (5'-CCTACGGGNGGCWGCAG-3') and S-D-Bact0785-a-A-21 (5'-GACTACHVGGGTATCTAATCC-3') for bacterial rRNA genes (Herlemann et al., 2011), and $\operatorname{arch} 349 \mathrm{~F}\left(5^{\prime}\right.$-GYGCASCAGKCGMGAAW-3') and arch806R (5'-GGACTACVSGGGTATCTAAT-3') for archaeal rRNA genes (Takai and Horikoshi, 2000). The $\mathrm{PCR}$ was carried out in a $50-\mu \mathrm{L}$ total volume formulated as follows: $10 \mu \mathrm{L}$ of $5 \times$ PrimeSTAR Buffer, $4 \mu \mathrm{L}$ of dNTP mixture (2.5 $\mathrm{m} M$ each), $0.5 \mu \mathrm{L}$ of PrimeSTAR HS DNA polymerase (Takara Bio Inc., Kusatsu, Japan), $1 \mu \mathrm{L}$ of each primer $(10 \mu M), 32.5 \mu \mathrm{L}$ of distilled $\mathrm{H}_{2} \mathrm{O}$, and $1 \mu \mathrm{L}$ of template DNA $(10 \mathrm{ng} / \mu \mathrm{L})$. The following PCR conditions were used: 30 cycles for bacteria and 40 cycles for archaea, with each cycle consisting of denaturation at $98^{\circ} \mathrm{C}(10 \mathrm{~s})$, annealing at $55^{\circ} \mathrm{C}(15 \mathrm{~s})$, and extension at $72^{\circ} \mathrm{C}(30 \mathrm{~s})$. Amplicons were used for the $2 \times 300$-bp sequencing reaction with the MiSeq Reagent Kit V3 (Caporaso et al., 2012). Data quality control and analyses were performed using the QIIME version 1.8.0 pipeline (Caporaso et al., 2010), and then singletons were removed. Operational taxonomic units were generated from sequences clustered at a 97\% similarity threshold using the UCLUST algorithm (Edgar, 2010). Chimeric sequences were removed from the analysis using the ChimeraSlayer algorithm (http: //microbiomeutil.sourceforge.net/\#A_CS). Taxonomy was assigned using the Greengenes database (version 13.8; McDonald et al., 2012) at a 90\% similarity threshold. Differences in biodiversity between control and WSCA-fed cultures were compared by alpha diversity metrics, including the following: Chao1, Shannon index, phylogenetic diversity whole tree, observed number of operational taxonomic units, and Good's coverage. All the sequences have been deposited in GenBank under the accession number DRA006952.

\section{Statistical Analysis}

The data from batch culture studies were averaged $(\mathrm{n}=4)$ for ANOVA using the GLM procedure of SPSS version 16.0 J (IBM, Tokyo, Japan). Tukey's method was used for multiple comparisons. Orthogonal contrasts were used to test linear, quadratic, and cubic effects of the dose level of WSCA. In the RUSITEC experiment, the data, including $\mathrm{pH}$, VFA, and ammonia, were subjected to ANOVA using the MIXED procedure of SPSS with repeated measures. The model included the effects of treatment, time after feeding, and the interaction of those 2 parameters, random effects of fermentors within treatments, and residual error. Time after feeding was used as the repeated measure. Unstructured covariance structure was used for the analysis. Data on gas and DM disappearance, qPCR, and next-generation sequencing (MiSeq) from samples taken at $0 \mathrm{~h}$ (before feeding) for $2 \mathrm{~d}$ from each fermentor were averaged and compared (control vs. WSCA) by Student's t-test. Multiple testing correction was used for qPCR and Miseq data using Bonferroni type. Statistical significance was inferred at $P<0.05$.

\section{RESULTS}

\section{Batch Culture}

Comparison of the potency of 2 different types of CA for modulating rumen fermentation is shown in Table 1. Gas production was not changed by supplementation with either CA compared with supplementation with the control cellulose. Supplementation with WSCA yielded increased total VFA concentration, acetate concentration, and molar proportion of acetate without affecting propionate or butyrate production. Supplementation with ISCA did not yield changes in the concentrations of any of these VFA. Microbial changes are shown in Figure 1. The absolute abundance of total bacteria, expressed as log copy number per milliliter of culture, was slightly decreased by ISCA but not by WSCA. However, the relative abundance of specific species and groups in the total bacteria showed significant changes: WSCA yielded increased abundance of genus Prevotella and P. ruminicola and decreased abundance of F. succinogenes, R. flavefaciens, and genus Treponema compared with the control. At the same time, ISCA yielded increased abundance of the Butyrivibrio group and decreased the abundance of $F$. succinogenes. Based on these results, we selected WSCA as the more potent form of CA for modulating rumen fermentation (for promoting acetate production) and microbiota; the characteristics of WSCA were then evaluated in the subsequent experiments.

The dose response of rumen fermentation to the selected WSCA is shown in Table 2. The production of total gas, $\mathrm{CO}_{2}$, total VFA, acetate, propionate, and butyrate was increased by supplementation with increasing dose levels of WSCA in all 3 relationships (linear, quadratic, and cubic). The best-fit response for these parameters except propionate production was in linear relationship according to the least $P$-values. We set the dose of WSCA to $15 \%$ of total feed for continuous culture evaluation in which more significant responses in fermentation, in particular the increase of acetate 
Table 1. Comparison of 2 different types of cellulose acetate (CA), insoluble CA (ISCA) and water-soluble CA (WSCA), in alterations of rumen fermentation

\begin{tabular}{lccccc}
\hline & \multicolumn{2}{c}{ Treatment $^{1}$} \\
\\
\cline { 3 - 4 } Parameter & Control & ISCA & WSCA & SEM & P-value \\
\hline Total gas, mL & 15.2 & 15.2 & 14.4 & 0.3 & 0.19 \\
$\mathrm{CO}_{2}, \mathrm{~mL}$ & 11.6 & 11.6 & 11.0 & 0.2 & 0.12 \\
$\mathrm{CH}_{4}, \mathrm{~mL}$ & 3.6 & 3.6 & 3.4 & 0.1 & 0.69 \\
$\mathrm{H}_{2}, \mathrm{~mL}$ & $\mathrm{ND}^{2}$ & $\mathrm{ND}$ & $\mathrm{ND}$ & - & - \\
Total VFA, mmol/L & $95.6^{\mathrm{b}}$ & $91.0^{\mathrm{b}}$ & $99.7^{\mathrm{a}}$ & 1.2 & $<0.01$ \\
Acetate, mmol/L & $60.1^{\mathrm{b}}$ & $58.0^{\mathrm{b}}$ & $64.9^{\mathrm{a}}$ & 1.0 & $<0.01$ \\
Propionate, mmol/L & $21.3^{\mathrm{ab}}$ & $19.8^{\mathrm{b}}$ & $22.1^{\mathrm{a}}$ & 0.3 & 0.01 \\
n-Butyrate, mmol/L & 9.5 & $9.0^{\mathrm{b}}$ & 9.0 & 0.0 & 1.00 \\
Acetate, molar \% & $63.3^{\mathrm{b}}$ & $63.8^{\mathrm{b}}$ & $65.1^{\mathrm{a}}$ & 0.2 & $<0.01$ \\
Propionate, molar \% & $22.5^{\mathrm{a}}$ & $21.7^{\mathrm{b}}$ & $22.2^{\mathrm{ab}}$ & 0.1 & 0.04 \\
n-Butyrate, molar \% & $9.7^{\mathrm{a}}$ & $9.8^{\mathrm{a}}$ & $9.1^{\mathrm{b}}$ & 0.1 & $<0.01$ \\
\hline
\end{tabular}

${ }_{\mathrm{a}, \mathrm{b}}$ Means within a row with different superscripts differ $(P<0.05)$.

${ }^{1}$ Supplementation of each substrate was set at $10 \%$ in final culture.

${ }^{2}$ Not detected.

production, can be expected with relevant microbial changes. This might provide mechanistic understanding of acetate increase in WSCA supplementation.

\section{Continuous Culture}

Rumen fermentation profiles and feed digestibility in RUSITEC are shown in Table 3. Total gas, $\mathrm{CH}_{4}$, and $\mathrm{CO}_{2}$ production were not affected, but a small amount of $\mathrm{H}_{2}$ accumulated when WSCA was added. Addition of WSCA did not change ruminal $\mathrm{pH}$ or total VFA concentration. However, the concentration and molar proportion of acetate increased in the presence of WSCA, whereas those of propionate decreased. No change was observed in butyrate production. Ammonia concentration increased with WSCA supplementation. Disappearance of DM, NDF, and ADF was not affected by the addition of WSCA. As observed in the batch

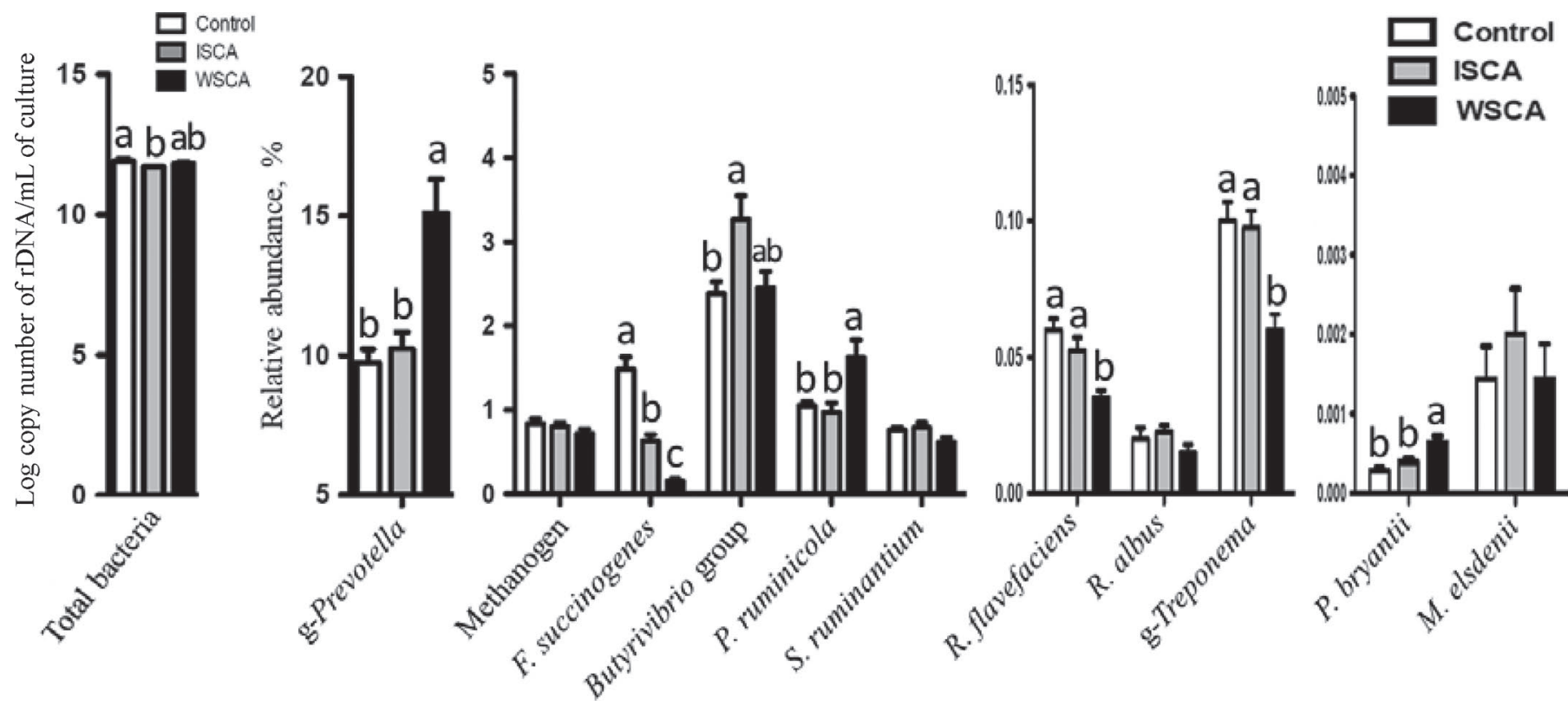

Figure 1. Relative abundance of rumen representative bacterial species and groups in batch culture as affected by insoluble cellulose acetate (ISCA) and water-soluble cellulose acetate (WSCA) supplementation. Each substrate was supplemented at 10\% in final culture. Quantified species and groups are genus Treponema (g-Treponema), genus Prevotella (g-Prevotella), total methanogens, Fibrobacter succinogenes, Butyrivibrio group, Ruminococcus flavefaciens, Ruminococcus albus, Prevotella ruminicola, Prevotella bryantii, Selenomonas ruminantium, and Megasphaera elsdenii. Letters $(\mathrm{a}-\mathrm{c})$ indicate statistical significance $(P<0.05)$. Error bars indicate SD. 
Table 2. Dose response of ruminal gas and VFA production to water-soluble cellulose acetate (WSCA) supplementation

\begin{tabular}{|c|c|c|c|c|c|c|c|c|c|}
\hline Parameter & \multicolumn{5}{|c|}{ Level of supplementation (\%) } & SEM & \multicolumn{3}{|c|}{ Contrast $^{1}$} \\
\hline Total gas, $\mathrm{mL}$ & 12.3 & 13.0 & 13.8 & 13.7 & 15.2 & 0.2 & $2.9 \times 10^{-6}$ & $2.1 \times 10^{-5}$ & $3.7 \times 10^{-5}$ \\
\hline $\mathrm{CH}_{4}, \mathrm{~mL}$ & 2.5 & 2.6 & 2.4 & 2.3 & 2.6 & 0.0 & 0.64 & 0.28 & 0.03 \\
\hline $\mathrm{H}_{2}, \mathrm{~mL}$ & $\mathrm{ND}^{2}$ & ND & ND & ND & ND & - & - & - & - \\
\hline Total VFA, mmol/L & 72.8 & 77.6 & 89.7 & 102.0 & 109.9 & 3.3 & $6.2 \times 10^{-12}$ & $8.6 \times 10^{-11}$ & $1.9 \times 10^{-10}$ \\
\hline n-Butyrate, mmol/L & 7.6 & 8.0 & 8.5 & 9.1 & 9.6 & 0.2 & $3.1 \times 10^{-8}$ & $3.3 \times 10^{-7}$ & $2.3 \times 10^{-6}$ \\
\hline
\end{tabular}

${ }^{1} P$-value for the linear, quadratic, and cubic effects of WSCA supplementation at $0,7.5,15,22.5$, and $30 \%$ in final culture.

${ }^{2}$ Not detected.

culture study, supplementation with WSCA yielded increased ruminal acetate production without negatively influencing feed digestion.

Microbial profiles with or without WSCA supplementation are shown in Figure 2. Absolute abundance of total bacteria, expressed as log copy number per milliliter of culture, did not change in the presence of WSCA. Supplementation with WSCA yielded increases in the relative abundance of genus Prevotella and $P$. ruminicola and decreases in the relative abundance of $F$. succinogenes, $R$. flavefaciens, $S$. dextrinosolvens, $R$. amylophilus, and $A$. lipolytica. The changes were especially apparent in the increases of genus Prevotella (15.1 vs. $42.2 \%)$ and $P$. ruminicola (0.4 vs. $2.7 \%$ ) and decreases of $F$. succinogenes (5.1 vs. $0.2 \%$ ).

Rumen microbiota were further analyzed by MiSeq and compared. The number of bacterial sequences passing the quality filter was 56,614 for control (minimum = $42,547$; median $=57,286)$ and 74,771 for the treatment

Table 3. Effect of water-soluble cellulose acetate (WSCA) supplementation on rumen fermentation profiles in rumen simulation technique cultures

\begin{tabular}{|c|c|c|c|c|}
\hline \multirow[b]{2}{*}{ Parameter } & \multicolumn{2}{|c|}{ Treatment $^{1}$} & \multirow[b]{2}{*}{ SEM } & \multirow[b]{2}{*}{$P$-value } \\
\hline & Control & WSCA & & \\
\hline Total gas, $\mathrm{mL}$ & 1,005 & 886.5 & 36.2 & 0.10 \\
\hline $\mathrm{CO}_{2}, \mathrm{~mL}$ & 887.1 & 788.0 & 31.3 & 0.12 \\
\hline $\mathrm{CH}_{4}, \mathrm{~mL}$ & 115.1 & 91.7 & 6.6 & 0.07 \\
\hline $\mathrm{H}_{2}, \mathrm{~mL}$ & 2.8 & 6.8 & 0.9 & $<0.01$ \\
\hline $\mathrm{pH}$ & 6.1 & 6.1 & 0.0 & 0.61 \\
\hline Total VFA, mmol/L & 87.8 & 89.2 & 2.2 & 0.77 \\
\hline Acetate, $\mathrm{mmol} / \mathrm{L}$ & 38.1 & 47.7 & 1.3 & $<0.01$ \\
\hline Propionate, $\mathrm{mmol} / \mathrm{L}$ & 30.8 & 23.4 & 1.0 & $<0.01$ \\
\hline n-Butyrate, $\mathrm{mmol} / \mathrm{L}$ & 10.4 & 10.7 & 0.3 & 0.57 \\
\hline Acetate, molar \% & 43.6 & 53.5 & 0.9 & $<0.01$ \\
\hline Propionate, molar \% & 34.8 & 26.2 & 0.8 & $<0.01$ \\
\hline n-Butyrate, molar \% & 12.0 & 12.0 & 0.1 & 0.68 \\
\hline Ammonia, $\mathrm{mg}$ of $\mathrm{N} / \mathrm{dL}$ & 4.3 & 9.7 & 0.7 & $<0.01$ \\
\hline DM digestibility, \% & 43.1 & 43.2 & 0.5 & 0.97 \\
\hline NDF digestibility, \% & 18.0 & 18.4 & 0.6 & 0.79 \\
\hline ADF digestibility, \% & 13.8 & 13.2 & 0.7 & 0.69 \\
\hline
\end{tabular}

${ }^{1}$ Each substrate was supplemented at $15 \%$ in final culture.
WSCA $($ minimum $=62,622 ;$ median $=69,158)$. For archaea, 35,908 sequences were obtained from samples of control ( minimum $=21,899$; median $=37,422)$, and 33,919 sequences were obtained for WSCA (minimum $=20,492$; median $=30,814)$. Changes in biodiversity indices inferred from bacterial and archaeal sequence reads are shown in Table 4. The high levels of Good's coverage (control vs. treatment, respectively: 98.9 vs. 98.8 for bacteria, 97.0 vs. 97.5 for archaea) suggest that obtained sequences represented the majority of bacteria and archaea present in the cultures. The total number of operational taxonomic units, Chao1, and phylogenetic diversity (PD_Whole_tree) for bacteria and archaea were not changed except that the bacterial Shannon index was lowered by supplementation with WSCA. Thus, almost all indices for biodiversity were not greatly affected by supplementation with WSCA. Changes in bacterial and archaeal communities at different taxonomic levels with WSCA supplementation are shown in Tables 5 and 6 . For bacteria at the phylum level, WSCA yielded increased abundance of Bacteroidetes, Planctomycetes, and Spirochaete and decreased abundance of Fibrobacteres, Firmicutes, and Verrucomicrobia. At the genus level, WSCA yielded increased abundance of Prevotella and Treponema and decreased abundance of Fibrobacter, Ruminococcus, and unclassified bacteria belonging to the Bacteroidales and Ruminococcaceae (see Supplemental Table S2 for other bacteria; https://doi.org/10.3168/jds.2018-14969). The archaeal community structure was not affected by WSCA supplementation at either the class or the genus level.

\section{DISCUSSION}

The in vitro results presented here are the first evidence (to our knowledge) to indicate that WSCA alters the rumen fermentation pattern toward higher acetate production without negatively affecting gas production and feed digestion (Tables 1-3). Because acetate is a 

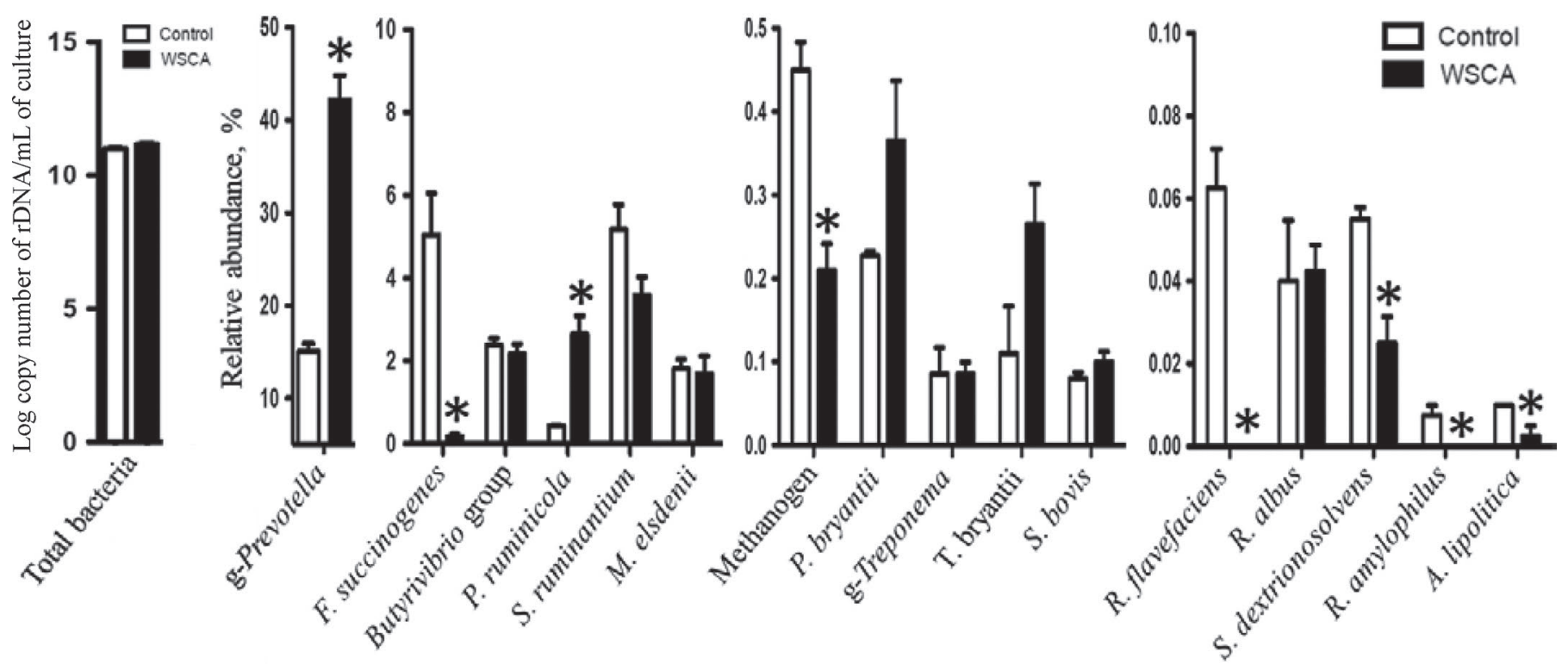

Figure 2. Relative abundance of rumen representative bacterial species and groups in rumen simulation technique as affected by water-soluble cellulose acetate (WSCA) supplementation. The WSCA was supplemented at $15 \%$ in total feed substrate. Quantified species and groups are genus Treponema (g-Treponema), genus Prevotella (g-Prevotella), total methanogens, Fibrobacter succinogenes, Butyrivibrio group, Ruminococcus flavefaciens, Ruminococcus albus, Prevotella ruminicola, Prevotella bryantii, Treponema bryantii, Succinivibrio dextrinosolvens, Ruminobacter amylophilus, Selenomonas ruminantium, Megasphaera elsdenii, Anaerovibrio lipolytica, and Streptococcus bovis. Asterisk (*) indicates statistical significance $(P<0.05)$. Error bars indicate SD.

major VFA supporting host animal nutrition, in particular fatty acid synthesis (Nafikov and Beitz, 2007), $\mathrm{CA}$ is expected to have potential for use as a feed or additive to promote the production of this important organic acid. The shift in rumen fermentation with CA observed in the present study is presumed to reflect primarily changes in the rumen microbial population (Figures 1 and 2; Table 5). Further analysis of this shift will need to focus on the pathways and rumen microbes

Table 4. Bacterial and archaeal biodiversity in rumen simulation technique cultures by MiSeq analysis (Illumina Inc., San Diego, CA) as affected by water-soluble cellulose acetate (WSCA) supplementation analysis of rumen simulation technique culture

\begin{tabular}{lccrrr}
\hline & \multicolumn{2}{c}{ Treatment $^{1}$} & & \\
\cline { 2 - 3 } Item & Control & WSCA & SEM & P-value \\
\hline Bacterial community & & & & \\
Operational taxonomic units & 3,040 & 2,978 & 143.0 & 0.30 \\
Chao1 & 6,152 & 6,598 & 283.5 & 0.49 \\
Shannon (H') & 7.9 & 7.6 & 0.1 & 0.01 \\
PD_whole_tree & 166 & 155 & 5.1 & 0.84 \\
Good's coverage, \% & 98.9 & 98.8 & 0.2 & 0.16 \\
Archaeal community & & & & \\
Operational taxonomic units & 770 & 764 & 49.2 & 0.95 \\
Chao1 & 1,371 & 1,295 & 92.7 & 0.71 \\
Shannon (H') & 2.5 & 2.9 & 0.1 & 0.10 \\
PD_whole_tree & 17.8 & 17.5 & 0.8 & 0.89 \\
Good's coverage, \% & 97.0 & 97.5 & 0.1 & 0.77 \\
\hline
\end{tabular}

${ }^{1}$ Each substrate was supplemented at $15 \%$ in final culture. responsible for CA degradation and acetate production. In this context, the difference in the molecular structures of WSCA and ISCA may provide a clue.

Notably, although WSCA affected rumen fermentation, ISCA did not (Table 1). This distinction in the effect on acetate production may reflect differences in the physicochemical characteristics of these 2 types of CA. An important factor could be the DS by acetyl group in the tested CA. The DS defines a physical property of CA (rendering the molecule soluble or insoluble) that is closely related to the accessibility of CA to CA-degrading enzymes such as esterases and glucanases (Altaner et al., 2003; Takeda et al., 2016). The conformation of CA (specifically, the presence and frequency of acetyl group substitutions) might also affect the formation of enzyme-substrate complexes. Previous studies have reported that WSCA (i.e., CA with a low DS) is more susceptible to enzymatic degradation than is ISCA (CA with a high DS; Reese, 1957; Puls et al., 2011). Acetyl esterase plays a key role in the release of acetyl groups from $\mathrm{CA}$ and in the degradation of the main cellulose chain of CA, yielding more acetate than does endoglucanase (Moriyoshi et al., 2003, 2013). Therefore, acetyl esterase is likely to be a critical enzyme in WSCA metabolism in the models tested here. Haske-Cornelius et al. (2017) reported that even the most active esterases belonging to the carbohydrate esterase family 2 could deacetylate only those CA with DS values of up to 1.8. 
Table 5. Effect of water-soluble cellulose acetate (WSCA) supplementation on rumen bacterial community in rumen simulation technique cultures evaluated by MiSeq (Illumina Inc., San Diego, CA) ${ }^{1}$

\begin{tabular}{|c|c|c|c|c|}
\hline \multirow[b]{2}{*}{ Relative abundance, $\%$} & \multicolumn{2}{|c|}{ Treatment $^{2}$} & \multirow[b]{2}{*}{ SEM } & \multirow[b]{2}{*}{$P$-value } \\
\hline & Control & WSCA & & \\
\hline \multicolumn{5}{|l|}{ Phylum level } \\
\hline Bacteroidetes & 29.5 & 40.5 & 1.3 & $<0.01$ \\
\hline Fibrobacteres & 9.7 & 0.4 & 1.6 & 0.01 \\
\hline Firmicutes & 45.7 & 37.3 & 1.5 & $<0.01$ \\
\hline Planctomycetes & 0.9 & 1.5 & 0.2 & 0.04 \\
\hline Spirochaetes & 1.9 & 4.5 & 0.9 & 0.03 \\
\hline Verrucomicrobia & 1.6 & 0.9 & 0.1 & $<0.01$ \\
\hline \multicolumn{5}{|l|}{ Genus level } \\
\hline Unclassified Bacteroidales & 9.2 & 5.4 & 0.6 & $<0.01$ \\
\hline Unclassified BS11 & 4.1 & 0.4 & 0.5 & $<0.01$ \\
\hline Prevotella & 13.9 & 32.5 & 1.5 & $<0.01$ \\
\hline Unclassified S24-7 & 0.9 & 1.6 & 0.3 & 0.04 \\
\hline CF231 & 1.0 & 0.4 & 0.1 & $<0.01$ \\
\hline Fibrobacter & 9.7 & 0.4 & 1.6 & $<0.01$ \\
\hline Unclassified Lachnospiraceae & 2.3 & 1.8 & 0.2 & 0.02 \\
\hline Butyrivibrio & 0.3 & 1.2 & 0.1 & 0.04 \\
\hline Unclassified Ruminococcaceae & 6.9 & 4.2 & 0.5 & $<0.01$ \\
\hline Ruminococcus & 1.9 & 0.3 & 0.4 & $<0.01$ \\
\hline Megasphaera & 3.8 & 5.5 & 0.1 & $<0.01$ \\
\hline Unclassfied Pirellulaceae & 0.9 & 1.5 & 0.9 & 0.03 \\
\hline Treponema & 1.7 & 4.4 & 0.2 & 0.04 \\
\hline Unclassfied $R F P 12$ & 1.1 & 0.7 & 0.9 & 0.02 \\
\hline
\end{tabular}

${ }^{1}$ Only taxa showing significant changes are indicated.

${ }^{2}$ Each substrate was supplemented at $15 \%$ in final culture.

Therefore, it can be postulated that various esterases produced by the rumen microbiota are capable of releasing acetate from WSCA $(\mathrm{DS}=0.78)$ but not from ISCA (DS $=2.4)$, an inference that is consistent with the results of the present study.

Although several kinds of esterases are capable of deacetylating CA, acetyl xylan esterases (AXE) have been shown to be particularly active in the degradation of CA (Biely, 2012). Altaner et al. (2003) reported that 8 AXE from different carbohydrate esterase families exhibited activity against CA with DS 0.7 and 1.4. Among rumen microbes, Prevotella encodes several AXE (Hespell and O'Bryan-Shah, 1988; Purushe et al., 2010). Gene expression of the carbohydrate esterases of $P$. ruminicola is highly induced when this species is grown on an ester-enriched substrate (Kabel et al., 2011). It is possible that $P$. ruminicola grows well, synthesizes more esterase, and releases more acetate during growth in the presence of WSCA. Therefore, bacteria of this group might play an important role in WSCA degradation in the rumen. In fact, the abundance of Prevotella was elevated with WSCA supplementation (compared with that in controls) in the present batch and continuous culture studies (Figures 1 and 2; Table 5). Growth stimulation of Prevotella also may yield increased ammonia production, given that microbes of this group are known to be involved in feed protein breakdown in the rumen (Wallace et al., 1997). Indeed, ammonia levels were elevated in WSCA-supplemented cultures in RUSITEC (Table 3 ).

Our pure culture study indicated that 3 Prevotella species, including P. ruminicola, Prevotella brevis, and Prevotella bryantii, grew more abundant in the presence of WSCA, yielding enhanced levels of acetate (unpublished results). These preliminary results support the hypothesis that Prevotella contributes to the increased production of acetate from WSCA. On the other hand, the observed decrease in abundance of some fibrolytics (including Fibrobacter and Ruminococcus) in WSCAsupplemented cultures (Figures 1 and 2; Table 5) cannot be fully explained. In fact, these decreases were apparent not only in relative abundance (Figures 1 and 2) but also in absolute abundance of those groups (data not shown). We postulate that alternative fibrolytic systems [comprising uncharacterized fibrolytic bacteria (Kobayashi, 2006) or unknown groups of Prevotella (Bekele et al., 2010)] may compensate for this decrease in the abundance of fibrolytic representatives given that fiber digestion was not affected by WSCA supplementation (Table 3). Prevotella is the predominant rumen bacterial group (Stevenson and Weimer, 2007), exhibiting genetic diversity (Ramsak et al., 2000) that correlates with the range of specific metabolic niches (Matsui et al., 2000; Bekele et al., 2010); therefore, much attention has been paid to the role of Prevotella in ruminant nutrition. The use of CA to "boost" the abundance of this bacterial group could provide a mechanism for alterations in the rumen microbiota while also providing a potential tool for exploring the nutritional function of Prevotella in the rumen.

Table 6. Effect of water-soluble cellulose acetate (WSCA) supplementation on rumen archaeal community in rumen simulation technique cultures evaluated by MiSeq (Illumina Inc., San Diego, CA)

\begin{tabular}{lrrrr}
\hline & \multicolumn{2}{c}{ Treatment $^{1}$} & & \\
\cline { 2 - 3 } Relative abundance, $\%$ & Control & WSCA & SEM & $P$-value \\
\hline Class level & 85.5 & 83.1 & 2.7 & 0.46 \\
Methanobacteria & 0.5 & 1.5 & 0.6 & 0.14 \\
Methanomicrobia & 2.3 & 4.0 & 0.9 & 0.12 \\
Thermoplasmata & 11.7 & 11.4 & 1.6 & 0.86 \\
Other & & & & \\
Genus level & 0.6 & 1.0 & 0.2 & 0.07 \\
Unclassified Methanobacteriaceae & 84.6 & 81.8 & 2.8 & 0.39 \\
Methanobrevibacter & 0.5 & 1.3 & 0.5 & 0.17 \\
Unclassified Methanomicrobiales & 2.3 & 4.0 & 0.9 & 0.12 \\
vadinCA11 & 12.0 & 12.0 & 1.5 & 0.99 \\
Other & & &
\end{tabular}

${ }^{1}$ Each substrate was supplemented at $15 \%$ in final culture. 
It will not be easy to determine precisely from where the observed acetate is derived. If all of the acetate originates from the acetyl groups of WSCA, a maximum of $80.0 \mu \mathrm{mol}$ of acetate per tube could have been released (based on the presence per tube of $0.02 \mathrm{~g}$ of WSCA with an assumed molecular weight of 194.9 and DS 0.78). In fact, net production of acetate from WSCA was $86.7 \mu \mathrm{mol}$ per tube (as calculated by subtracting acetate in the blank tubes from acetate in the treated tubes). Thus, the acetate generated in the treated samples presumably derived not only from the acetyl groups of WSCA but also from the main cellulose chain of WSCA or components of the basal feed substrate. Therefore, WSCA can serve as a useful substrate for promoting acetate production via the activation of specific groups of rumen microbes, positively contributing to ruminant nutrition. However, the contribution of CA to acetate production from basal substrate is to be further confirmed in a more detailed study to validate the small difference of acetate production as used in the above calculation.

Acetate is known to be a major substrate for milk fat synthesis (Jacobs et al., 2013; Urrutia and Harvatine, 2017). The present study suggests that WSCA may serve as a ruminal acetate enhancer and therefore that WSCA should be considered as a candidate feed or additive for use in dairy cattle. For instance, milk fat production has been reported to decrease under heat stress conditions (Das et al., 2016); supplementation with WSCA may be an option for addressing this problem. In addition, we need to consider the suitable dose of CA needed for practical applications. The WSCA yielded dose-dependent increases in total VFA and acetate production, suggesting that supplementation at doses exceeding $15 \%$ may be needed for significant effect (Table 2). The RUSITEC study confirmed the effectiveness of supplementation at a $15 \%$ level, though a practical feeding level of WSCA is to be recommended for further applications after assessment in in vivo studies.

Because CA is synthesized by using cellulose and chemicals, pure materials are expensive (US $\$ 2.99 / \mathrm{kg}$ : Trade Statistics of Japan, 2017) and thereby unrealistic in feed use. However, plenty of residue in the industrial process goes to waste management (Malone, 1990), though the exact amount of CA waste is not available in statistics. Such waste could be used as animal feed in the future once functionality of the pure material as tested in the present study is successfully evaluated. Although the present evaluation is performed with in vitro systems, the outcome can be useful for gaining insight and information about $\mathrm{CA}$ as a candidate feed supplement.

\section{CONCLUSIONS}

The results of the present study suggest that WSCA could be a potent feed supplement for modifying the rumen microbiota and fermentation without adverse effects on feed digestion. We found that ruminal acetate production was increased by supplementation with WSCA, an effect that may reflect increased growth of Prevotella. However, the microbial shift caused by WSCA may differ depending on the primary diet of the animals; further investigations using feeding experiments will be needed to draw more general conclusions. Furthermore, given that various types of CA with different DS and degree of polymerization can be synthesized enzymatically, additional work will be needed to experimentally identify the best CA material for use in ruminants.

\section{ACKNOWLEDGMENTS}

This study was supported in part by Grant-in-Aid for Challenging Exploratory Research to Y. K. (no. 16K15020) from the Ministry of Education, Culture, Sports, Science and Technology, Tokyo, Japan.

\section{REFERENCES}

Altaner, C., B. Saake, M. Tenkanen, J. Eyzaquirre, C. B. Faulds, P. Biely, L. Viikari, M. Siika-aho, and J. Puls. 2003. Regioselective deacetylation of cellulose acetates by acetyl xylan esterases of different CE-families. J. Biotechnol. 105:95-104.

Bekele, A. Z., S. Koike, and Y. Kobayashi. 2010. Genetic diversity and diet specificity of ruminal Prevotella revealed by $16 \mathrm{~S}$ rRNA genebased analysis. FEMS Microbiol. Lett. 305:49-57.

Bergman, E. N. 1990. Energy contributions of volatile fatty acids from the gastrointestinal tract in various species. Physiol. Rev. 70:567-590.

Biely, P. 2012. Microbial carbohydrate esterases deacetylating plant polysaccharides. Biotechnol. Adv. 30:1575-1588.

Bouraoui, R., M. Lahmar, A. Majdoub, M. Djemali, and R. Belyea. 2002. The relationship of temperature-humidity index with milk production of dairy cows in a Mediterranean climate. Anim. Res. 51:479-491.

Candido, R. G., and A. R. Gonçalves. 2016. Synthesis of cellulose acetate and carboxymethylcellulose from sugarcane straw. Carbohydr. Polym. 152:679-686.

Caporaso, J. G., J. Kuczynski, J. Stombaugh, K. Bittinger, F. D. Bushman, E. K. Costello, N. Fierer, A. G. Peña, J. K. Goodrich, J. I. Gordon, G. A. Huttley, S. T. Kelley, D. Knights, J. E. Koenig, R. E. Ley, C. A. Lozupone, D. McDonald, B. D. Muegge, M. Pirrung, J. Reeder, J. R. Sevinsky, P. J. Turnbaugh, W. A. Walters, J. Widmann, T. Yatsunenko, J. Zaneveld, and R. Knight. 2010. QIIME allows analysis of high-throughput community sequencing data. Nat. Methods 7:335-336.

Caporaso, J. G., C. L. Lauber, W. A. Walters, D. Berg-Lyons, J. Huntley, N. Fierer, S. M. Owens, J. Betley, L. Fraser, M. Bauer, N. Gormley, J. A. Gilbert, G. Smith, and R. Knight. 2012. Ultrahighthroughput microbial community analysis on the Illumina HiSeq and MiSeq platforms. ISME J. 6:1621-1624.

Czerkawski, J. W., and G. Breckenridge. 1977. Design and development of a long-term rumen simulation technique (Rusitec). Br. J. Nutr. 38:371-384 
Das, R., L. Sailo, N. Verma, P. Bharti, J. Saikia, Imtiwati, and R. Kumar. 2016. Impact of heat stress on health and performance of dairy animals: A review. Vet. World 9:260-268.

Edgar, R. C. 2010. Search and clustering orders of magnitude faster than BLAST. Bioinformatics 26:2460-2461.

Fei, Z., S. Huang, J. Yin, F. Xu, and Y. Zhang. 2015. Preparation and characterization of bio-based degradable plastic films composed of cellulose acetate and starch acetate. J. Polym. Environ. 23:383-391.

Flint, H. J. 1997. The rumen microbial ecosystem - Some recent developments. Trends Microbiol. 5:483-488.

Gaana, S., L. Mauclaire, P. Ruer, V. Salimova, T. Tran, and M. Heuberger. 2011. Thermal degradation of cellulose acetate in presence of bis-phosphoramidates. J. Anal. Appl. Pyrolysis 90:33-41.

Haske-Cornelius, O., A. Pellis, G. Tegl, S. Wurz, B. Saake, L. Roland, S. Andries, G. S. Nyanhongo, and M. G. Guebitz. 2017. Enzymatic systems for cellulose acetate degradation. Catalysts 7:287. https:// doi.org/10.3390/catal7100287.

Herlemann, D. P. R., M. Labrenz, K. Jurgens, S. Bertilsson, J. J. Waniek, and A. F. Andersson. 2011. Transition in bacterial communities along the $2000 \mathrm{~km}$ salinity gradient of the Baltic Sea. ISME J. 5:1571-1579.

Hespell, R. B., and P. J. O'Bryan-Shah. 1988. Esterase activities in Butyrivibrio fibrisolvens strains. Appl. Environ. Microbiol. 54:1917-1922

Ishigaki, T., W. Sugano, M. Ike, and M. Fujita. 2000. Enzymatic degradation of cellulose acetate plastic by novel degrading bacterium Bacillus sp. S2055. J. Biosci. Bioeng. 90:400-405.

Jacobs, A. A. A., J. Dijkstra, J. S. Liesman, M. J. VandeHaar, A. L. Lock, A. M. van Vuuren, W. H. Hendriks, and J. van Baal. 2013. Effects of short- and long-chain fatty acids on the expression of stearoyl-CoA desaturase and other lipogenic genes in bovine mammary epithelial cells. Animal 7:1508-1516.

Kabel, M. A., C. J. Yeoman, Y. Han, D. Dodd, C. A. Abbas, J. A. M. Bont, M. Morrison, I. K. O. Cann, and R. I. Mackie. 2011. Biochemical characterization and relative expression levels of multiple carbohydrate esterases of the xylanolytic rumen bacterium Prevotella ruminicola 23 grown on an ester-enriched substrate. Appl. Environ. Microbiol. 77:5671-5681.

Kobayashi, Y. 2006. Inclusion of novel bacteria in rumen microbiology: Need for basic and applied science. Anim. Sci. J. 77:375-385.

Koike, S., H. Yabuki, and Y. Kobayashi. 2007. Validation and application of real-time polymerase chain reaction assays for representative rumen bacteria. Anim. Sci. J. 78:135-141.

Lalia, B. S., V. Kochkodan, R. Hashaikeh, and N. Hilal. 2013. A review on membrane fabrication: Structure, properties and performance relationship. Desalination 326:77-95.

Malone, N. H. 1990. Eastman Chemical Company's leadership role in waste management. Inter. Fiber J. June:30-32.

Matsui, H., K. Ogata, K. Tajima, M. Nakamura, T. Nagamine, R. I. Aminov, and Y. Benno. 2000. Phenotypic characterization of polysaccharidases produced by four Prevotella type strains. Curr. Microbiol. 41:45-49.

McDonald, D., M. N. Price, J. Goodrich, E. P. Nawrocki, T. Z. DeSantis, A. Probst, G. L. Andersen, R. Knight, and P. Hugenholtz. 2012. An improved Greengenes taxonomy with explicit ranks for ecological and evolutionary analyses of bacteria and archaea. ISME J. 6:610-618.

McDougall, E. I. 1948. Studies on ruminant saliva. 1. The composition and output of sheep's saliva. Biochem. J. 43:99-109.

Miyamoto, T., Y. Sato, T. Shibata, M. Tanahashi, and H. Inagaki. 1985. ${ }^{13} \mathrm{C}$-NMR spectral studies on the distribution of substituents in water-soluble cellulose acetate. J. Polym. Sci. A Polym. Chem. 23:1373-1381.

Moriyoshi, K., D. Koma, H. Yamanaka, K. Sakai, and T. Ohmoto. 2013. Expression and characterization of a thermostable acetylxylan esterase from Caldanaerobacter subterraneus ssp. tengcongensis involved in the degradation of insoluble cellulose acetate. Biosci. Biotechnol. Biochem. 77:2495-2498.
Moriyoshi, K., T. Ohmoto, T. Ohe, and K. Sakai. 2003. Role of endo1,4- $\beta$-glucanases from Neisseria sicca SB in synergistic degradation of cellulose acetate. Biosci. Biotechnol. Biochem. 67:250-257.

Nafikov, R. A., and D. C. Beitz. 2007. Carbohydrate and lipid metabolism in farm animals. J. Nutr. 137:702-705.

Puls, J., S. A. Wilson, and D. Holter. 2011. Degradation of cellulose acetate-based materials: A review. J. Polym. Environ. 19:152-165.

Purushe, J., D. E. Fouts, M. Morrison, B. A. White, R. I. Mackie, North American Consortium for Rumen Bacteria, P. M. Coutinho, B. Henrissat, and K. E. Nelson. 2010. Comparative genome analysis of Prevotella ruminicola and Prevotella bryantii: Insights into their environmental niche. Microb. Ecol. 60:721-729.

Ramsak, A., M. Peterka, K. Tajima, J. C. Martin, J. Wood, E. A Johnston, R. I. Aminov, J. H. Flint, and G. Avgustin. 2000. Unraveling the genetic diversity of ruminal bacteria belonging to the CFB phylum. FEMS Microbiol. Ecol. 33:69-79.

Reese, E. T. 1957. Biological degradation of cellulose derivatives. Ind Eng. Chem. 49:89-93.

Robertson, R. M., W. C. Thomas, J. N. Suthar, and D. M. Brown 2012. Accelerated degradation of cellulose acetate cigarette filters using controller-release acid catalysis. Green Chem. 14:2266-2272.

Sakai, K. T. Yamauchi, F. Nasaku, and T. Ohe. 1996. Biodegradation of cellulose acetate by Neisseria sicca. Biosci. Biotechnol. Biochem. 60:1617-1622.

Samios, E., R. K. Dart, and J. V. Dawkins. 1997. Preparation, characterization and biodegradation studies on cellulose acetates with varying degrees of substitution. Polymer (Guildf.) 38:3045-3054

Shimamoto, S., S. Okada, H. Taniguchi, and T. Nakamura. 2014. Cellulose acetate fiber, cellulose acetate fiber molded article, and methods respectively for producing said cellulose acetate fiber and said cellulose acetate fiber molded article. Patent no. WO2015107565A1.

Stevenson, D. M., and P. J. Weimer. 2007. Dominance of Prevotella and low abundance of classical ruminal bacterial species in the bovine rumen revealed by relative quantification real-time PCR. Appl. Microbiol. Biotechnol. 75:165-174.

Takai, K., and K. Horikoshi. 2000. Rapid detection and quantification of members of the archaeal community by quantitative PCR using fluorogenic probes. Appl. Environ. Microbiol. 66:5066-5072.

Takeda, N., Y. Enomoto-Rogers, A. Takemura, and T. Iwata. 2016. Synthesis and enzymatic degradation of randomly substituted 2,3,6-O-cellulose acetate and regioselectively substituted 2,3-Ocellulose acetate. Polym. Degrad. Stabil. 129:125-132.

Teramoto, Y. 2015. Functional thermoplastic materials from derivatives of cellulose and related structural polysaccharides. Molecules 20:5487-5527.

Trade Statistics of Japan. 2017. Appendix of trade statistics by product line. Accessed Aug. 16, 2018. http://www.customs.go.jp/ toukei/sankou/code/code.htm.

Urrutia, N. L., and K. J. Harvatine. 2017. Acetate dose-dependently stimulates milk fat synthesis in lactating dairy cows. J. Nutr. 147:763-769.

Wallace, R. J., R. Onodera, and M. A. Cotta. 1997. Metabolism of nitrogen-containing compounds. Pages $238-328$ in The Rumen Microbial Ecosystem. 2nd ed. P. N. Hobson and C. S. Stewart, ed Springer, London, UK.

Watanabe, Y., R. Suzuki, S. Koike, K. Nagasima, M. Mochizuki, R. J. Forster, and Y. Kobayashi. 2010. In vitro evaluation of cashew nut shell liquid as a methane-inhibiting and propionate-enhancing agent for ruminants. J. Dairy Sci. 93:5258-5267.

Weatherburn, M. W. 1967. Phenol-hypochlorite reaction for determination of ammonia. Anal. Chem. 39:971-974.

Yu, Z., and M. Morrison. 2004. Improved extraction of PCR-quality community DNA from digesta and fecal samples. Biotechniques $36: 808-812$.

Zhou, X., X. Lin, K. L. White, S. Lin, H. Wu, S. Cao, L. Huang, and L. Chen. 2016. Effect of the degree of substitution on the hydrophobicity of acetylated cellulose for production of liquid marbles. Cellulose 23:811-821. 